\title{
Broadband High-Gain Beam-Scanning Antenna Array for Millimeter-Wave Applications
}

\author{
Chun-Xu Mao, Steven Gao, and Yi Wang
}

\begin{abstract}
A novel method of achieving low-profile, broadband microstrip array antennas with high antenna gain is proposed for millimeter-wave (mm-wave) applications. The element employs a novel $3^{\text {rd }}$-order vertically coupled resonant structure that a U-slot resonator in the ground is used to couple with the feeding resonator and the radiating patch, simultaneously. This proposed structure can significantly improve the bandwidth and frequency selectivity without increasing the thickness of the antenna. Then, to achieve the subarray, a new wideband power divider with loaded resonators is employed, which can be used to further improve the bandwidth. To demonstrate the working schemes of broadside radiation and scanned beam, two $4 \times 4$ array antennas are implemented on the same board. Measured results agree well with the simulations, showing a wide bandwidth from 22 to $32 \mathrm{GHz}(\mathrm{FBW}=37 \%)$ with the gain of around 19 dBi. The beam scanning array can realize a scanning angle of over 25 degrees over a broadband. In addition, due to the filtering features are integrated in the design, the proposed antenna could also reduce the complexity and potential cost of the frontends.
\end{abstract}

Index Terms- Broadband, beam scanning, filtering, millimeter-wave, antenna array, antenna, $5 \mathrm{G}$.

\section{INTRODUCTION}

The emerging fifth-generation (5G) mobile communication has attracted intensive research interests in academia and industry because of its huge potentials such as high data rate and significant reduction of digital traffic [1]-[2]. In the 5G era, lots of things such as electronic devices, vehicles and the equipment in the offices and homes will be wirelessly connected through the Internet. Users will be able to access ultra-high-definition (UHD) multimedia streaming and services such as Virtual Reality (VR) and Augmented Reality (AR) [2]. All these potential services will inevitably demand a very wide bandwidth to support the extremely high data rate in $5 \mathrm{G}$ wireless communication. The millimeter wave ( $\mathrm{mm}$-wave) frequency band is widely believed to be a good candidate to realize a wideband operation.

For mm-wave applications, other problems to be concerned are the higher transmission loss and link stability, which could be overcome by increasing the gain and adopting the adaptive directional beam [2]. Massive MIMO base station is a promising technique for improving the capacity and service quality by accurately concentrating the transmitted energy to the mobile users [3]-[5], as the architecture shown in Fig. 1. The massive MIMO antenna has multiple antenna subarrays in two dimensions and each can adaptively direct the beams to the users in azimuth and elevation directions. Thanks to each subarray is composed of $n \times n$ radiation elements, high antenna gain and steerable beam can be achieved. Due to the short wavelength at mm-waves and limited coverage of each $5 \mathrm{G}$ cellular, the mm-wave base-station antennas with low cost, low profile and light weight will be in huge demand in urban areas.

To date, several mm-wave antennas have been reported for $5 \mathrm{G}$ communications. In [6], a stacked patch was proposed to achieve a

Manuscript submitted on April 1, 2017; This work is supported by UK EPSRC grant EP/N032497/1 and YW is supported by UK EPSRC under Contract EP/M013529/1.

C. X. Mao and S. Gao are with School of Engineering and Digital Arts, University of Kent, UK (cm688@kent.ac.uk).

Y. Wang is with the Department of Engineering Science, University of Greenwich, UK.

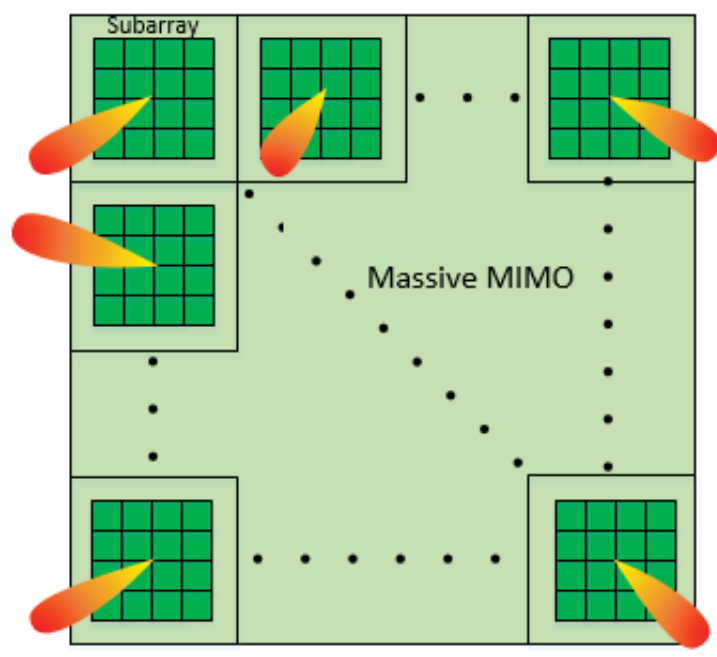

Fig. 1. Architecture of the massive MIMO base-station antenna array.

broadband antenna at $28 \mathrm{GHz}$. But this antenna suffers from poor radiation that some radiation nulls emerged in the main-lobe. In [7], a tilted antenna was designed by combining a patch and a waveguide aperture, but which is not suitable for integration. Dual-band (28/38 $\mathrm{GHz}$ ) antenna element with circular polarization was reported in [8]. However, the bandwidths are considerable narrow (less than 3\%). Normally, bandwidth can be enhanced by adding parasitic patches, but it will lead to the high profile and cost [9]-[10]. In [11]-[12], mm-wave antenna arrays using substrate integrated waveguide (SIW) and low temperature co-fired ceramic (LTCC) technologies were achieved. In [13]-[15], massive MIMO arrays for 5G wireless communications were reported. However, these works cannot fulfill the requirements of broad bandwidth, high gain, low profile and low cost, simultaneously.

In this communication, a broadband, high gain microstrip array antenna with low profile is proposed. The antenna is achieved by employing a novel vertically coupled resonant structure, which could effectively broaden the bandwidth of a microstrip antenna without increasing the thickness of the antenna. In addition, the proposed antenna exhibits good frequency selectivity and out-of-band rejection performance due to the integrated resonant elements in the design. These features could lead to the removal of the mm-wave band-pass filters and $50 \Omega$ interfaces, and therefore the complexity and the potential cost could be significantly reduced [16]-[23]. To demonstrate the operation schemes, a $4 \times 4$ antenna array with broadside radiation and a $4 \times 4$ beam scanning array are respectively implemented.

\section{IMPLEMENTATION}

\section{A. Antenna element and its topology}

Fig. 2(a) shows the configuration of the proposed microstrip antenna element. It consists of two stacked substrates. The patch is printed on the top layer of the upper substrate whereas the microstrip line and hairpin resonator are printed on the bottom layer of the lower substrate. The patch and the feeding network share the same ground plane, which is placed on the top layer of the lower substrate. In the ground plane, a U-shaped slot is etched to form a slot resonator. The 


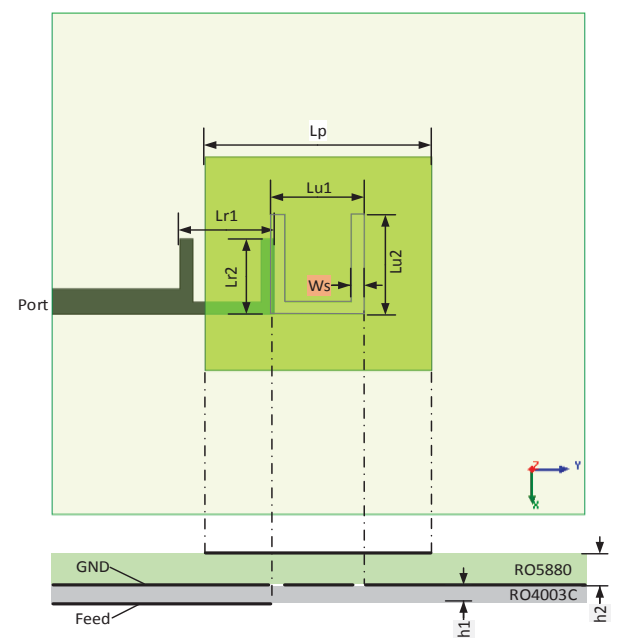

(a)

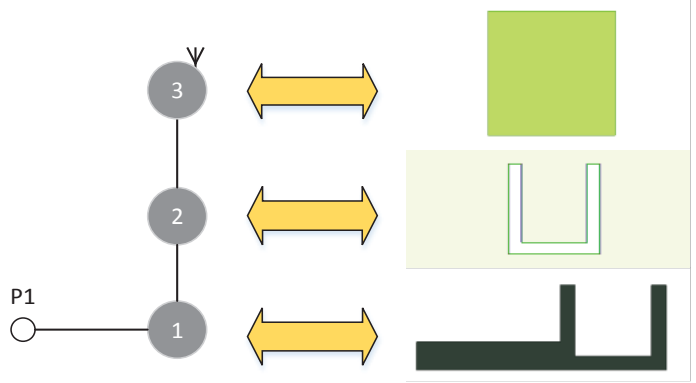

(b)

Fig. 2. (a) Configurations of the proposed antenna element, (b) equivalen circuit. $L p=3.05 \mathrm{~mm}, h 1=0.2 \mathrm{~mm}, h 2=0.787 \mathrm{~mm}, L r 1=1.3 \mathrm{~mm}, L r 2=1.15$ $\mathrm{mm}, \mathrm{Lu} 1=1.2 \mathrm{~mm}, \mathrm{Lu} 2=1.4 \mathrm{~mm}$, Ws $=0.2 \mathrm{~mm}$.

patch, U-slot resonator and the hairpin resonator are stacked and vertically coupled, shaping a high-order filtering antenna. In this work, RO 4003C substrate with a permittivity of 3.55 and loss tangent of 0.0027 is used for the lower substrate. To reduce the antenna loss, RO 5880 substrate with a permittivity of 2.2 and loss tangent of 0.0009 is used for the upper substrate. The thicknesses of the RO $4003 \mathrm{C}$ and the RO 5880 are $0.2 \mathrm{~mm}$ and $0.787 \mathrm{~mm}$, and thus the total thickness of the antenna is less than $1 \mathrm{~mm}$. The optimization was performed using high frequency structural simulator (HFSS 15) and the optimized parameters are presented in the caption of Fig. 2.

To illustrate the design approaches, the antenna element is decomposed by each layer and shown in Fig. 2(b). The hairpin resonator on the bottom layer is connected by a $50 \Omega$ microstrip line, which serves as the $1^{\text {st }}$-order resonator. The U-shaped slot in the ground plane is served as the $2^{\text {nd }}$-order resonator. The U-slot is a half-wavelength resonator and its resonance can be controlled by adjusting the length and the width of the slot. The square patch on the top layer, not only works the radiation element but also the last order resonator of the antenna. The patch, U-slot resonator, and hairpin resonator are stacked and coupled in vertical, shaping a 3rd-order filtering antenna. All of them have the same resonant frequency at around $27 \mathrm{GHz}$. The dimensions of the U-slot and hairpin resonators can be approximately evaluated using the expressions below,

$$
\begin{aligned}
& L u 1+2 \cdot \operatorname{Lu} 2 \approx \frac{c}{2 f_{0} \sqrt{\varepsilon_{\text {eff } 1}}} \\
& \operatorname{Lr} 1+2 \cdot \operatorname{Lr} 2 \approx \frac{c}{2 f_{0} \sqrt{\varepsilon_{\text {eff } 2}}}
\end{aligned}
$$

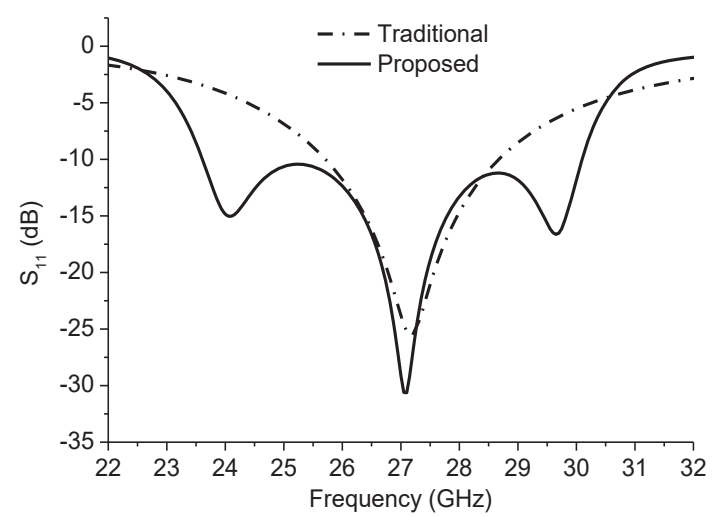

Fig. 3. Comparison of the simulated $\mathrm{S}_{11}$ between the proposed antenna element and traditional patch.

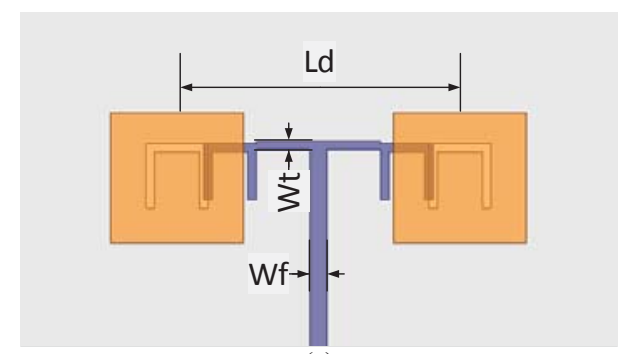

(a)

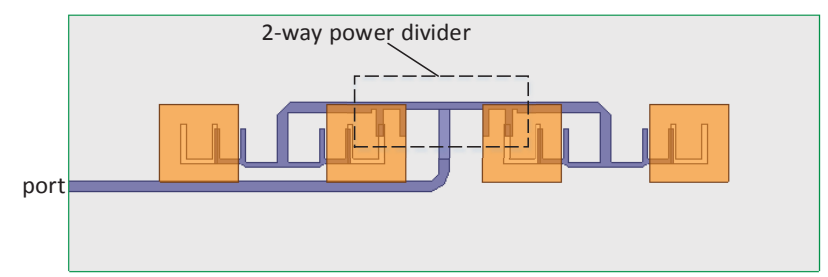

(b)

Fig. 4. Configurations of the proposed subarrays: (a) $1 \times 2$ subarray, subarray-I; (b) $1 \times 4$ subarray, subarray-II. $W f=0.4 \mathrm{~mm}, W t=0.24 \mathrm{~mm}, L d=$ $6 \mathrm{~mm}$.

where, $f_{0}$ is the resonant frequency. $\varepsilon_{\text {eff }} 1$ and $\varepsilon_{\text {eff }} 2$ are the effective dielectric constants of the slot-line in the ground and the microstrip, respectively. $c$ is the speed of light in free space.

Compared with traditional patch antennas, the proposed vertically coupled antenna could significantly broaden the impedance bandwidth of the patch antenna. Fig. 3 compares the simulated $S_{11}$ of a traditional aperture feed patch antenna and the proposed antenna with the same thickness. As can be observed, the traditional patch has only one resonant frequency at $27.2 \mathrm{GHz}$, shaping a bandwidth from 26 to 28.5 $\mathrm{GHz}(\mathrm{FBW}=9.3 \%)$. In contrast, the proposed antenna has three matching points at around 24, 27 and $30 \mathrm{GHz}$, respectively, generating a broad bandwidth from 23.5 to $30.5 \mathrm{GHz}(\mathrm{FBW}=26 \%)$. In addition, the proposed antenna exhibits an improved $3^{\text {rd }}$-order filtering feature.

\section{B. Subarrays}

To meet the requirements of high gain in mm-wave applications while providing the beam scanning ability, the antenna elements are combined to form the antenna subarrays. Fig. 4(a) shows the configurations of the proposed $1 \times 2$ subarray (denoted as subarray-I). The distance between the elements is set to be $6 \mathrm{~mm}, 0.54$ wavelength at $27 \mathrm{GHz}$. In this work, due to the symmetry of the design, the elements can be excited from both sides while maintaining a consistent phase, as shown in Fig. 4(a). Such a layout is beneficial to reduce the size and complexity of the feeds. Fig. 4(b) shows the configuration of 


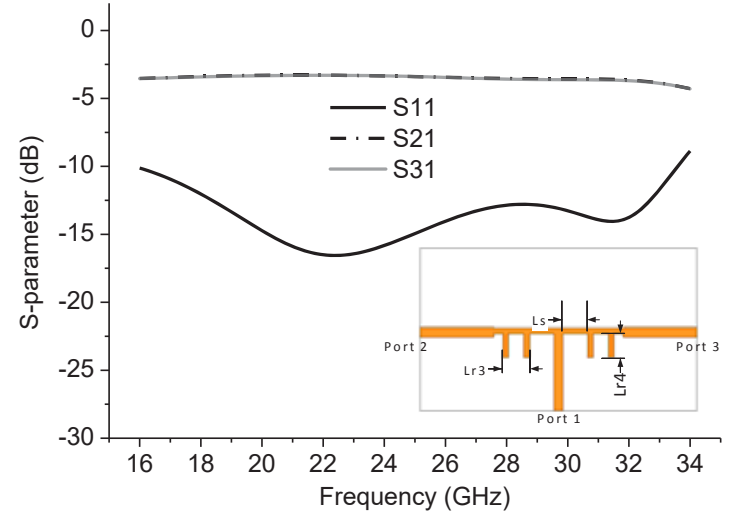

Fig. 5. Configuration and simulated S-parameters of the two-way power divider. $L s=1.5 \mathrm{~mm}, L 3=1.1 \mathrm{~mm}, L 4=1.2 \mathrm{~mm}$.

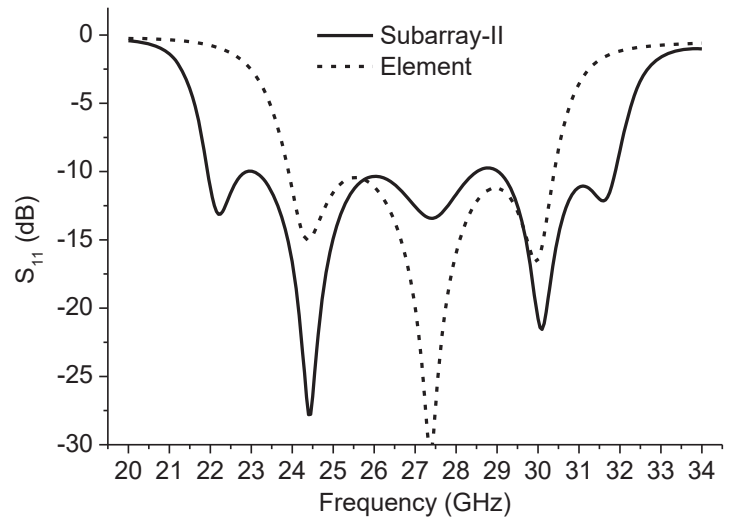

(a)

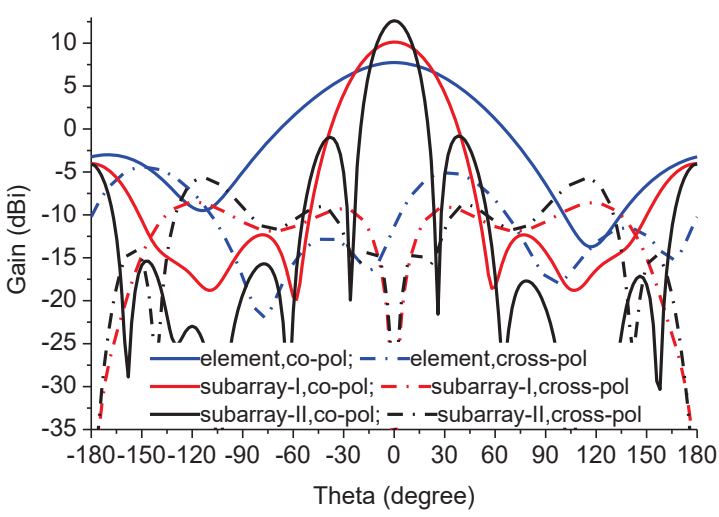

(b)

Fig. 6. Simulated results comparisons between the element and the subarrays (a) S-parameters, (b) radiation patterns.

the $1 \times 4$ subarray (denoted as subarray-II), which is combined by two $1 \times 2$ subarrays and fed using a two-way power divider. Different from traditional power dividers, the adopted divider integrates two resonant structures at its two outputs, which can further enhance the impedance bandwidth of the antenna.

Fig. 5 shows the simulated S-parameters of the power divider. The results show that it has a good power divider performance over a very wide bandwidth with two reflection nulls emerged at around 22 and 32 $\mathrm{GHz}$, respectively.

Fig. 6 compares the simulated S-parameters and radiation patterns of the proposed element and the two subarrays. As can be seen from Fig. 6(a), there are totally three resonant frequencies for the element

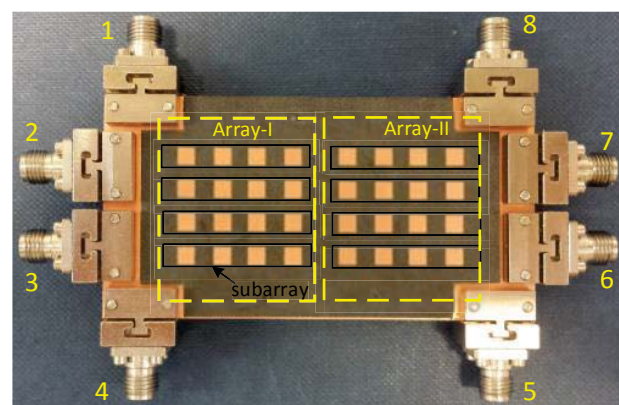

(a)

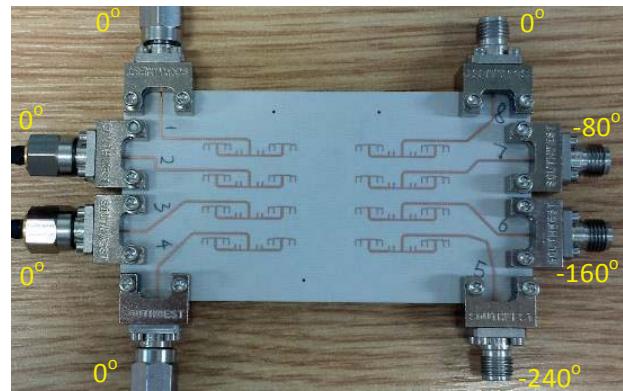

(b)

Fig. 7. Prototype of the two arrays: (a) front view, (b) bottom view

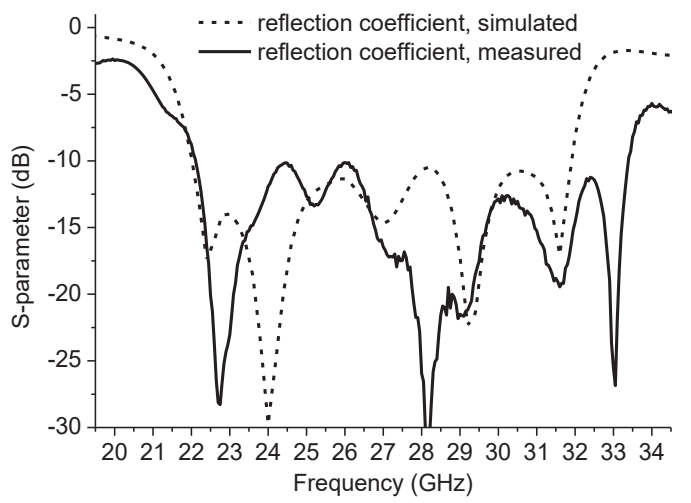

Fig. 8. Simulated and measured reflection coefficients of the subarrays

but five resonant frequencies for the subarray-II. These two additional resonant frequencies result from the power divider presented in Fig. 5, which results in a wider impedance bandwidth from 22 to $32 \mathrm{GHz}$ $(\mathrm{FBW}=37 \%)$. The radiation patterns of the antenna element, subarray-I and II in Fig. 6(b) show that the three antennas have the maximum radiation in the broadside direction. The gains of the three antennas are $7.5 \mathrm{dBi}, 10 \mathrm{dBi}$ and $12.5 \mathrm{dBi}$, respectively.

\section{ANTENNA ARRAYS AND RESUltS}

Based on the $1 \times 4$ subarray, two $4 \times 4$ array antennas were conceived to demonstrate the operation schemes in Fig. 1. The first one is a regular array with the maximum radiation in the broadside direction, denoted as Array-I. The Array-I has the identical input phase and amplitude for each subarray. The second one is a beam scanning array, which has the gradient input phases for the four subarrays, denoted Array-II. Both arrays were fabricated on the same board, as the prototype shown in Fig. 7. To facilitate the measurement, a 4-way power divider was used to feed the subarrays.

Fig. 8 shows the simulated and measured reflection coefficients of the subarray. The measured results agree reasonably well with the simulations, showing a very wide impedance bandwidth from 22 to 

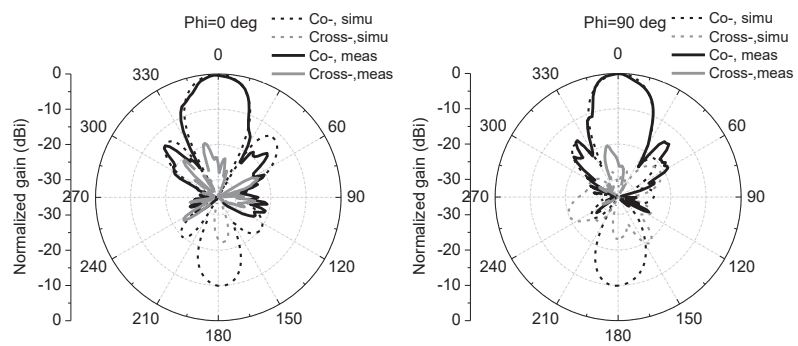

(a)
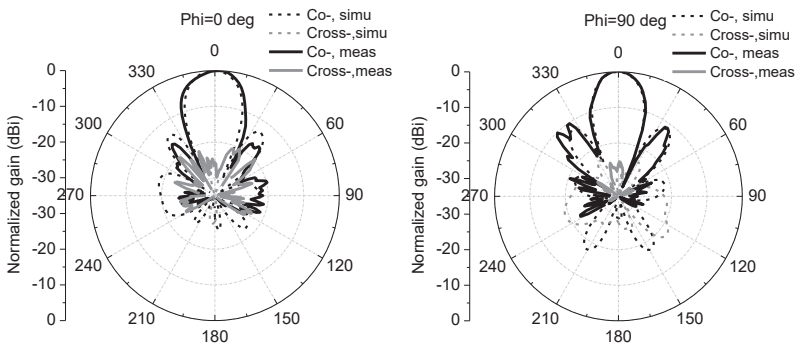

(b)
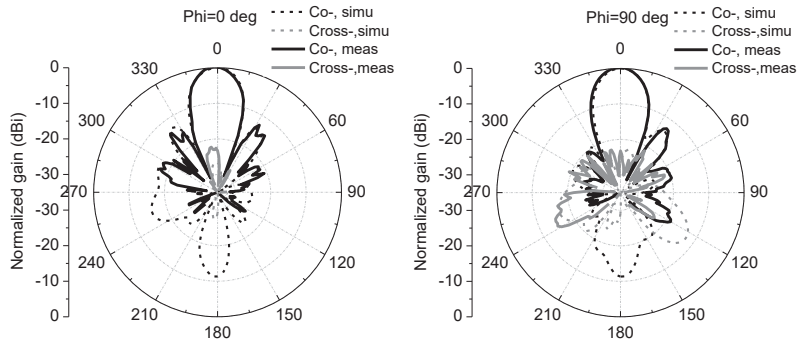

Fig. 9. Simulated and measured normalized E-plane and H-plane radiation patterns of the Array-I: (a) $23.5 \mathrm{GHz}$, (b) $27 \mathrm{GHz}$ and (c) $30.5 \mathrm{GHz}$.

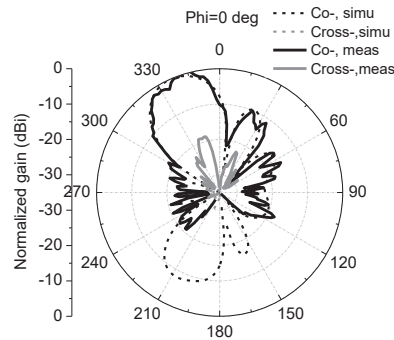

(a)

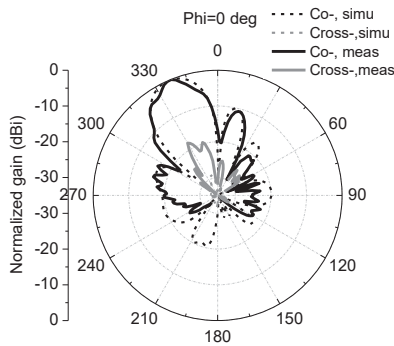

(c)

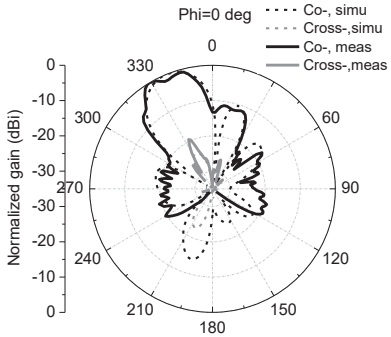

(b)

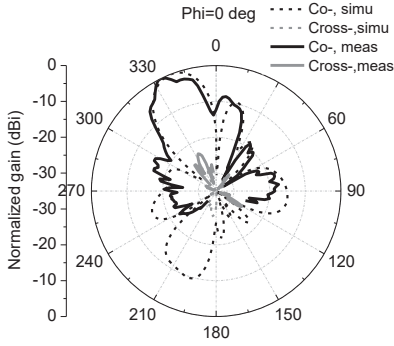

(d)
Fig. 10. Simulated and measured normalized radiation patterns of the Array-II: (a) $22.5 \mathrm{GHz}$, (b) $25.5 \mathrm{GHz}$, (c) $28.5 \mathrm{GHz}$ and (d) $31.5 \mathrm{GHz}$

$33.5 \mathrm{GHz}(\mathrm{FBW}=41.8 \%)$. Such a broad bandwidth is attributed to the proposed vertically coupled structure and the broadband power divider. The discrepancy between the simulations and measurements, especially the additional matching point at $33 \mathrm{GHz}$, may be caused by the unknown harmonics and fabrication tolerance.

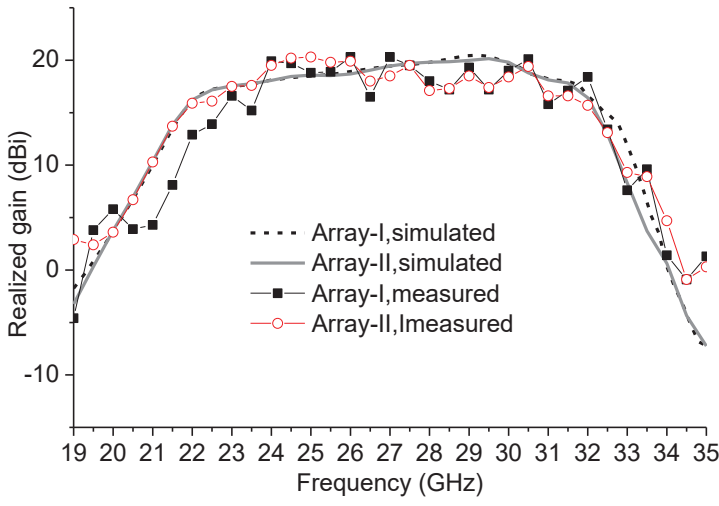

Fig. 11. Simulated and measured antenna gains of the Array-I and II.

Fig. 9 shows the simulated and measured normalized E- and H-plane radiation patterns of the Array-I at 23.5, 27 and $30.5 \mathrm{GHz}$, respectively. As can be seen, good agreement between the measurements and simulations is achieved. The Array-I exhibits an almost consistent broadside radiation over a broadband. The measured XPD in broadside direction is over $25 \mathrm{~dB}$. The minor discrepancy between the simulated and measured results is attributed to the measurement tolerance.

Fig. 10 shows the simulated and measured E-plane normalized radiation patterns of the Array-II at 22.5, 25.5, 28.5 and $31.5 \mathrm{GHz}$, respectively. The measured results of the Array-II agree reasonably well with the simulations, showing a scanned beam of over 25 degrees over a broadband. The measured side-lobes are below to $-10 \mathrm{~dB}$ and the XPD in the main beam are over $20 \mathrm{~dB}$.

Fig. 11 shows the simulated and measured realized gains of the Array-I and Array-II, respectively. As can be observed, both arrays exhibit a flat gain response of around $19 \mathrm{dBi}$ from 23 to $32 \mathrm{GHz}$. The measured results agree reasonably well with the simulations, except for some fluctuation of the measurements. Beyond the operation band, the gain drops dramatically to below $0 \mathrm{dBi}$, exhibiting a good out-of-band rejection performance.

\section{CONCLUSION}

In this communication, a novel broadband, low-profile microstrip array antenna with integrated filtering features is proposed for mm-wave applications. The technique contribution of this work includes: (1) a vertically coupled high-order resonant structure is employed to design low-profile broadband microstrip array antennas; (2) a novel broadband power divider is used to further improve the bandwidth and frequency selectivity of the antenna. To demonstrate the typical operation schemes, a $4 \times 4$ broadside radiation array and a 4 $\times 4$ beam scanning array were investigated and prototyped. Both the measured results agree well the simulations, demonstrating the proposed antenna is suitable for high-gain, mm-wave applications. Furthermore, the integrated filtering characteristics could reduce the complexity and potential cost of the RF front-end.

\section{REFERENCES}

[1] T. Rappaport, S. Sun, R. Mayzus, H. Zhao, Y. Azar, K. Wang, G. Wong, J. Schulz, M. Samimi and F. Gutierrez, "Millimeter wave mobile communications for $5 \mathrm{G}$ cellular: It will work!," IEEE Access, vol. 1, pp. 335-349, 2013.

[2] Samsung. (2015, Aug.). Samsung demo: 5G vision, Korea. Available: http://www.samsung.com/global/business/networks/insights/news/samsu ng-electronics-sets-5g-speed-record-at-7-5 gbpsover-30-times-faster-than -4 g-lte.

[3] F. Rusek, D. Persson, B. Lau, E. Larsson, T. Marzetta, O. Edfors and F. Tufvesson, "Scaling up MIMO: Opportunities and challenges with very 
large arrays," IEEE Signal Process. Mag., vol. 30, no. 1, pp. 40-60, Jan. 2013.

[4] F. Rusek, D. Persson, B. K. Lau, E. G. Larsson, T. L. Marzetta, O. Edfors, and F. Tufvesson, "Scaling up mimo: Opportunities and challenges with very large arrays,” IEEE Signal Process. Mag., vol. 30, no. 1, pp. 40-60, Jan. 2013.

[5] E. Larsson, O. Edfors, F. Tufvesson, and T. Marzetta, "Massive mimo for next generation wireless systems," IEEE Commun. Mag., vol. 52, no. 2, pp. 186-195, Feb. 2014

[6] S. Ershadi, A. Keshtkar, A. Abdelrahman, X. Yu and H. Xin, "Design of wideband unit-cell element for 5G antenna arrays," 2015 Asia-Pacific Microwave Conference (APMC), pp. 1-3, 2015.

[7] J. S. Park, J. B. Ko, H. K. Kwon, B. S. Kang, B. Park and D. Kim, “A tilted combined beam antenna for $5 \mathrm{G}$ communications using a $28-\mathrm{GHz}$ Band," IEEE Antennas Wireless Propog. Lett, vol. 15, pp. 1685-1688, 2016.

[8] H. Aliakbari, A. Abdipour, R. Mirzavand, A. Costanzo and P. Mousavi, "A single feed dual-band circularly polarized millimeter-wave antenna for 5G communication," 2016 10th European Conference on Antennas and Propag. (EuCAP), pp. 1-5, 2016.

[9] S. Gao and A. Sambell, "Low-Cost Dual-Polarized Printed Array with Broad Bandwidth,” IEEE Trans. Antennas Propag., vol. 52, no. 12, pp 3394-3397, Dec. 2004

[10] Y. X. Guo, K. M. Luk, and K. F. Lee, "Broadband dual-polarization patch element for cellular-phone base stations," IEEE Trans. Antennas Propag., vol. 50, no. 2, pp. 251-253, Feb. 2002.

[11] W. Zhai, V. Miraftab, M. Repeta, D. Wessel and W. Tong, "Dual-band millimeter-wave interleaved antenna array exploiting low-cost PCB technology for high speed 5G communication," 2016 IEEE MTT-S International Microw. Symposium (IMS), pp. 1-4, 2016.

12] F. F. Manzillo, M. Ettorre, M. Lahti, K. Kautio, D. Lelaidier, E. Seguenot, R. Sauleau, "A multilayer LTCC solution for integrating $5 \mathrm{G}$ access point antenna modules," IEEE Trans. Microw. Theory Techn., vol. 64, no. 7 , pp. 2272-2283, 2016.

[13] J. Hirokawa, "Millimeter-wave antenna technologies for 5G mobile communication systems," 2016 IEEE International Workshop on Electromagnetics: Applications and Student Innovation Competition (iWEM), pp. 1-3, 2016.

[14] M. M. Ali, A. R. Sebak, "Design of compact millimeter wave massive MIMO dual-band $(28 / 38 \mathrm{GHz})$ antenna array for future 5G communication systems," 2016 17th International Symposium on Antenna Technology and Applied Electromagnetics (ANTEM), pp. 1-2, 2016.

[15] Y. Gao, R. Ma, Y. Wang, Q. Zhang and C. Parini, "Stacked patch antenna with dual-polarization and low mutual coupling for massive MIMO," IEEE Trans. Antennas and Propag., to be published.

[16] C. X. Mao, S. Gao, Y. Wang, F. Qin and Q. X. Chu, "Multi-mode resonator-fed dual polarized antenna array with enhanced bandwidth and selectivity," IEEE Trans. Antennas and Propag., vol. 63, no. 12, pp. 5492-5499, Dec. 2015.

[17] C. X. Mao, S. Gao, Y. Wang, B. Sanz, Z. P. Wang, F. Qin and Q. X. Chu, J. Li, G. Wei, J. Xu, "Dual-band filtering-antenna with controllable bandwidth and harmonics suppression," IEEE Trans. Antennas and Propag., vol. 64, no. 9, pp. 4074-4077, Sep. 2016.

[18] X. Y. Zhang, W. Duan, Y. M. Pan, "High-gain filtering patch antenna without extra circuit," IEEE Trans. Antennas Propag., vol. 63, no. 12, pp. 5883-5888, Dec. 2015.

[19] Y. M. Pan, P. F. Hu, X. Y. Zhang and S.Y. Zheng, "A low profile high gain and wideband filtering antenna with metasurface," IEEE Trans. Antennas Propag., vol. 64, no. 5, pp. 2010-2016, May, 2016.

[20] Y. Zhang, X. Y. Zhang, L. H. Ye, Y. M. Pan, "Low-profile dual-band filtering patch antenna and its application to LTE MIMO system," IEEE Trans. Antennas Propag., vol. 65, no. 1, pp. 103-113, Jan, 2017.

[21] C. X. Mao, S. Gao, Y. Wang, F. Qin and Q. X. Chu, "Compact highly integrated planar duplex antenna for wireless communications," IEEE Trans. Microw. Theory Techn., vol. 64, no. 7, pp. 2006-2013, Jul. 2016.

[22] C. K. Lin and S. J. Chung, "A Filtering Microstrip Antenna Array," IEEE Trans. Microw. Theory Techn., vol. 59, no. 11, pp. 2856-2863, Mar. 2011.

[23] C. X. Mao, S. Gao, Y. Wang, Z. Wang, F. Qin, B. Sanz, Q. X. Chu, "An integrated filtering antenna array with high selectivity and harmonics suppression," IEEE Trans. Microw. Theory Techn., vol. 64, no. 6, pp. 1798-1805, Apr. 2016. 